\title{
Impact on Clinical Outcomes of Periodic Leg Movements During Sleep in Hospitalized Patients Following Acute Decompensated Heart Failure
}

\author{
Shoichiro Yatsu, MD; Takatoshi Kasai, MD, PhD; Shoko Suda, MD; Hiroki Matsumoto, MD; \\ Nanako Shiroshita, BSc; Mitsue Kato; Fusae Kawana, BSc; Azusa Murata, MD; \\ Takao Kato, MD; Masaru Hiki, MD; Hiroyuki Daida, MD
}

\begin{abstract}
Background: Periodic leg movements during sleep (PLM) are characterized by regularly recurring movement of the legs during sleep. Although PLM is common and a predictor of death in patients with chronic heart failure, the clinical significance of PLM in hospitalized patients with a reduced left ventricular ejection fraction (LVEF) following acute decompensated heart failure (ADHF) remains unknown.

Methods and Results: After initial improvement of acute signs and symptoms of ADHF, 94 consecutive patients with reduced LVEF who underwent polysomnography were enrolled. They were divided into 2 groups based on the presence or absence of severe PLM defined as PLM index $\geq 30$. The risks for clinical events, composite of all-cause death and rehospitalization, were assessed using a stepwise multivariable Cox proportional model including variables showing $\mathrm{P}<0.10$ in univariate analyses. Severe PLM was observed in 21 patients (22\%). At a median follow-up of 5.2 months, 30 patients experienced clinical events (32\%). In the multivariable analysis, the presence of severe PLM was significantly associated with increasing clinical events (hazard ratio, 2.16; 95\% confidence interval, 1.03-4.54; $P=0.042$ ) independent of hemoglobin level and the severity of sleep-disordered breathing.
\end{abstract}

Conclusions: In hospitalized patients with systolic dysfunction following ADHF, severe PLM was prevalent and significantly associated with increased risk of death and/or rehospitalization.

Key Words: Polysomnography; Sleep; Systolic heart failure

A lthough effective treatments for heart failure (HF) have been recently developed, it remains a major concern in terms of increasing hospitalization and mortality rates. ${ }^{1}$ Episodes of worsening of $\mathrm{HF}$, which usually manifest as acute decompensated HF (ADHF), lead to hospitalization and also progression of HF itself, possibly through alterations in cardiovascular physiology and systemic or cardiac metabolic conditions. ${ }^{2,3}$ Thus, hospitalization for ADHF should be identified as a specific entity or risk factor that contributes to the progression of HF. ${ }^{4,5}$ Consequently, among patients hospitalized for $\mathrm{ADHF}$, the identification of potential risk factors that are associated with increased risk of readmission and death is considered an important strategy in comprehensive HF care and may provide benefits by reducing readmission and mortality rates. ${ }^{68}$ One such approach may include identification of periodic leg movements during sleep (PLM).

PLM is characterized by regularly recurring movements of the legs during sleep, affecting $4-8 \%$ of the general population.9,10 Patients with chronic HF frequently have PLM. ${ }^{11-14}$ In addition, in patients with chronic HF, PLM can be a predictor of all-cause and cardiac death in those with either a reduced left ventricular ejection fraction (LVEF) or with preserved LVEF. ${ }^{\mathbf{1 4}, 15}$ However, the effect of PLM in hospitalized patients with reduced LVEF following an episode of ADHF remains to be elucidated. The aim of the present study was to evaluate the relationship between PLM in hospitalized patients with reduced LVEF following ADHF and clinical outcomes such as readmission and/or death.

\section{Methods}

Subjects

We enrolled 94 consecutive patients who were hospitalized between May 2012 and May 2014 at the Juntendo University

Received September 26, 2016; revised manuscript received November 19, 2016; accepted December 14, 2016; released online January 17, 2017 Time for primary review: 21 days

Department of Cardiovascular Medicine, Juntendo University School of Medicine, Tokyo (S.Y., T. Kasai, S.S., H.M., A.M., T. Kato, M.H., H.D.); Cardiovascular Respiratory Sleep Medicine, Juntendo University Graduate School of Medicine, Tokyo (T. Kasai, S.S., H.M., N.S., M.K., F.K.), Japan

Mailing address: Takatoshi Kasai, MD, PhD, Department of Cardiovascular Medicine, Juntendo University School of Medicine, Cardiovascular Respiratory Sleep Medicine, Juntendo University Graduate School of Medicine, 2-1-1 Hongo, Bunkyo-ku, Tokyo 113-8421, Japan. E-mail: kasai-t@mx6.nisiq.net

ISSN-1346-9843 All rights are reserved to the Japanese Circulation Society. For permissions, please e-mail: cj@j-circ.or.jp 
Hospital for ADHF in association with LV systolic dysfunction (defined as LVEF $<50 \%$ on echocardiography) and who underwent overnight polysomnography regardless of symptoms or signs of sleep disorders. ADHF was defined according to modified Framingham criteria. ${ }^{16}$ The exclusion criteria were acute coronary syndrome and cardiac surgery during the previous 4 weeks, end-stage renal disease requiring dialysis, cerebrovascular disease with neurological deficits, life-threatening malignancy, apparent obstructive lung disease as demonstrated by a forced expiratory volume $<70 \%$ in $1 \mathrm{~s} /$ forced vital capacity, and known sleep-disordered breathing (SDB). The study protocol was approved by the Juntendo University Hospital Institutional Review Board, and the study complied with the Declaration of Helsinki. Informed consent was given by all patients.

\section{Polysomnography}

All patients underwent overnight polysomnography using a digital polygraph system (Alice PDX, Philips Respironics Inc., Murrysville, PA, USA) for a few days after the initial improvement in the acute signs and symptoms of ADHF. Generally accepted definitions and scoring methods were used. ${ }^{17}$ Thoracoabdominal motion was monitored via respiratory inductance plethysmography, and airflow was measured by an oronasal thermal airflow sensor and nasal pressure cannula. Oxyhemoglobin saturation $\left(\mathrm{SO}_{2}\right)$ was monitored by oximetry. Apneas and hypopneas were quantified, and the severity of SDB was assessed using the frequency of apneas and hypopneas per hour of sleep (i.e., apnea-hypopnea index, AHI). For leg movements, electromyographic recordings were made from the anterior tibialis muscles of both legs. PLM was defined and scored using standard criteria from the American Academy of Sleep Medicine Manual for the Scoring of Sleep and Associated Events: a series of 4 consecutive leg movements lasting $0.5-5 \mathrm{~s}$ with an amplitude $\geq 8 \mu \mathrm{V}$ above the resting baseline and separated by intervals of 5-90s. ${ }^{17}$ A leg movement was not scored if it occurred during a period from $0.5 \mathrm{~s}$ preceding an apnea, hypopnea, or respiratory event-related arousal to $0.5 \mathrm{~s}$ following such an event. The PLM index (PLMI) was quantified as the frequency of PLMs per hour of sleep. In this study, patients were divided into 2 groups based on the presence or absence of severe PLM (i.e., PLMI $<30 / \mathrm{h}$ or $\geq 30 / \mathrm{h}$ ). ${ }^{17}$ The PLM-related arousal index (PLMAI) was also quantified as the frequency of PLM-related arousals per hour of sleep.

\section{Other Baseline Data and Outcome Data Collection}

On the same day of polysomnography, anthropometric data were obtained, and the presence or absence of atrial fibrillation (AF) and the functional status of the patients were determined according to ECG and the New York Heart Association (NYHA) classification system, respectively. Blood pressure (BP) and heart rate were measured just before the functional status assessment. Blood samples were obtained in the early morning after polysomnography was performed. The estimated glomerular filtration rate (eGFR) was calculated using the Modification of Diet in Renal Disease equation with a Japanese coefficient from baseline serum creatinine levels. ${ }^{18}$ Complete $2 \mathrm{D}$ echocardiography and Doppler ultrasound examination were performed using standard techniques on the day of blood sampling. The LVEF was calculated according to the modified Simpson method.

All patients were followed-up in hospital from the date of polysomnography until November 2014; outcome data were obtained by reviewing the hospital medical records in which all deaths and readmission events were recorded. The endpoint in the present study was a composite clinical outcome of death and readmission for ADHF. Readmission for ADHF was defined as the first unscheduled readmission to the cardiology ward with progressive symptomatic and/ or hemodynamic deterioration.

\section{Statistical Analysis}

To compare baseline characteristics between the 2 groups, $\chi^{2}$ test or Fisher's exact test for categorical variables and Student's t-test or Mann-Whitney U-test for continuous variables were used. The event-free survival curves were established using the Kaplan-Meier method and compared using the log-rank test. Univariable and multivariable Cox proportional hazards regression analyses were performed to evaluate the association between the presence of severe PLM and clinical outcomes. In the univariable analysis, the presence of severe PLM was used as an independent variable along with the following baseline variables: age, sex, body mass index, ischemic etiology, presence or absence of AF, implantable cardioverter defibrillator, cardiac resynchronization therapy, LVEF, systolic and diastolic BP, heart rate, eGFR, hemoglobin level, serum levels of sodium and potassium and $\mathrm{C}$-reactive protein (CRP), plasma B-type natriuretic peptide (BNP) level, medication use, other sleep study parameters (total sleep time, percentage of slow wave sleep, percentage of rapid eye movement [REM] sleep, mean and minimum $\mathrm{SO}_{2}$, PLMAI $[<$ or $\geq 5 / \mathrm{h}],{ }^{19}$ and AHI) and initiation of SDB treatment. Next, variables that showed $\mathrm{P}<0.10$ in the univariable analysis were entered into a multivariable forward stepwise Cox proportional hazards regression analysis with $\mathrm{P}<0.05$ to enter and $\mathrm{P} \geq 0.10$ to remove. The natural log-transformed values were used for CRP, BNP, and AHI, as these values were skewed. The assumption of proportional hazards was assessed using a log-minus-log survival graph. All these analyses were performed using a statistical software package (SPSS ver.23.0, IBM Corp., Armonk, NY, USA).

\section{Results}

Of the 94 patients, $21(22 \%)$ had severe PLM. The baseline characteristics of the patients with and those without severe PLM are shown in Table 1. Patients with severe PLM had a greater NYHA functional class, higher levels of plasma BNP and serum CRP, but lower hemoglobin levels compared with those without severe PLM. They were less likely to take angiotensin-converting enzyme inhibitors or angiotensin-receptor blockers. However, there were no significant differences between the 2 groups for the other baseline characteristics. Polysomnographic parameters are listed in Table 2. Patients with severe PLM had less REM sleep but greater arousal index and PLMAI than those without severe PLM. Overall, 18 patients were started on treatment for SDB, including 1 of those with severe PLM and 17 of those without severe PLM (4.8\% vs. 23.3\%, $\mathrm{P}=0.112$ ).

The outcome data were collected for a median duration of 5.2 (interquartile range, 8.1 ) months. During this period, 30 clinical events $(32 \%)$ occurred, of which $13(62 \%)$ were in patients with PLM, and $17(18 \%)$ in those without severe PLM. The cumulative event-free survival curves of these 2 groups are shown in the Figure. Patients with severe PLM 


\begin{tabular}{|c|c|c|c|}
\hline & $\begin{array}{c}\text { PLMI }<30 / h \\
(n=73)\end{array}$ & $\begin{array}{c}\mathrm{PLMI} \geq 30 / \mathrm{h} \\
(\mathrm{n}=21)\end{array}$ & $P$ value \\
\hline Age, years & $62.2 \pm 13.3$ & $66.8 \pm 14.9$ & 0.181 \\
\hline Female sex, n (\%) & $23(31.5)$ & $6(28.6)$ & 0.977 \\
\hline $\mathrm{BMI}, \mathrm{kg} / \mathrm{m}^{2}$ & $23.8 \pm 5.3$ & $23.2 \pm 4.6$ & 0.620 \\
\hline NYHA class & $2[1]$ & $3[1]$ & 0.004 \\
\hline II, n (\%) & $46(63.0)$ & $6(28.6)$ & 0.011 \\
\hline$\geq \mathrm{III}, \mathrm{n}(\%)$ & 27 (37.9) & $15(71.4)$ & \\
\hline Ischemic etiology, $\mathrm{n}(\%)$ & $22(30.1)$ & $9(42.9)$ & 0.407 \\
\hline $\mathrm{AF}, \mathrm{n}(\%)$ & $17(23.3)$ & $4(19.0)$ & 0.909 \\
\hline Diabetes mellitus, $\mathrm{n}(\%)$ & $17(23.3)$ & $8(38.1)$ & 0.283 \\
\hline $\mathrm{ICD}, \mathrm{n}(\%)$ & $4(5.5)$ & $2(9.5)$ & 0.613 \\
\hline CRT, n (\%) & $2(2.7)$ & $3(14.3)$ & 0.072 \\
\hline Systolic BP, mmHg & $106.9 \pm 18.7$ & $104.1 \pm 15.2$ & 0.533 \\
\hline Diastolic BP, mmHg & $60.7 \pm 10.9$ & $56.9 \pm 8.9$ & 0.148 \\
\hline Heart rate, beats/min & $69.4 \pm 10.3$ & $73.4 \pm 9.5$ & 0.570 \\
\hline LVEF, \% & $31.4 \pm 7.8$ & $29.8 \pm 9.4$ & 0.441 \\
\hline Hemoglobin, g/dL & $14.1 \pm 2.4$ & $12.4 \pm 2.2$ & 0.004 \\
\hline eGFR, $\mathrm{mL} / \mathrm{min} / 1.73 \mathrm{~m}^{2}$ & $60.47 \pm 23.0$ & $50.7 \pm 25.4$ & 0.101 \\
\hline Sodium, $\mathrm{mmol} / \mathrm{L}$ & $139.3 \pm 3.8$ & $138.8 \pm 3.8$ & 0.615 \\
\hline Potassium, mmol/L & $4.4 \pm 0.4$ & $4.4 \pm 0.4$ & 0.709 \\
\hline $\mathrm{CRP}, \mathrm{mg} / \mathrm{dL}$ & $0.2[0.4]$ & $0.6[1.2]$ & 0.026 \\
\hline $\mathrm{BNP}, \mathrm{pg} / \mathrm{mL}$ & $266.6[407.0]$ & $697.1[825.3]$ & 0.003 \\
\hline$\beta$-blockers, n (\%) & $67(91.8)$ & $18(85.7)$ & 0.413 \\
\hline ACEIs/ARBs, n (\%) & $67(91.8)$ & $14(66.7)$ & 0.010 \\
\hline Aldosterone blockers, n (\%) & $47(64.4)$ & $16(76.2)$ & 0.735 \\
\hline Loop diuretics, n (\%) & $60(82.2)$ & $18(85.7)$ & 0.961 \\
\hline
\end{tabular}

Data are expressed as mean \pm SD or median [interquartile range] for continuous variables and number (\%) for nominal variables. ACEI, angiotensin-converting enzyme inhibitors; ADHF, acute decompensated heart failure; AF, atrial fibrillation; ARB, angiotensin II receptor blockers; BMI, body mass index; BNP, B-type natriuretic peptide; BP, blood pressure; CRP, C-reactive protein; CRT, cardiac resynchronization therapy; eGFR, estimated glomerular filtration rate; ICD, implantable cardioverter defibrillator; LVEF, left ventricular ejection fraction; NYHA, New York Heart Association; PLM, periodic leg movement during sleep; PLMI, periodic leg movement index.

\begin{tabular}{|c|c|c|c|}
\hline & $\begin{array}{c}\text { PLMI }<30 / h \\
(n=73)\end{array}$ & $\begin{array}{c}\mathrm{PLMI} \geq 30 / \mathrm{h} \\
(\mathrm{n}=21)\end{array}$ & $P$ value \\
\hline Total sleep time, min & $367.5 \pm 89.0$ & $332.2 \pm 120.2$ & 0.144 \\
\hline$\%$ of slow wave sleep, $\%$ of TST & $10.2 \pm 8.3$ & $9.0 \pm 7.1$ & 0.555 \\
\hline$\%$ of REM sleep, $\%$ of TST & $16.4 \pm 6.4$ & $11.0 \pm 7.3$ & 0.001 \\
\hline Arousal index, events/h of sleep & 18.9 [19.3] & $30.3[21.9]$ & 0.015 \\
\hline $\mathrm{AHI}$, events/h of sleep & 22.7 [35.2] & 29.4 [32.4] & 0.327 \\
\hline Mean $\mathrm{SO}_{2}, \%$ & $96.0 \pm 1.7$ & $95.6 \pm 1.5$ & 0.355 \\
\hline Minimum $\mathrm{SO}_{2}, \%$ & $85.1 \pm 8.5$ & $85.0 \pm 7.9$ & 0.945 \\
\hline PLMI, events/h of sleep & $0.8[6.7]$ & $59.9[57.0]$ & $<0.001$ \\
\hline PLMAI, events/h of sleep & $0.2[1.0]$ & $6.0[12.2]$ & $<0.001$ \\
\hline
\end{tabular}

Data are expressed as mean \pm SD or as median [interquartile range]. AHI, apnea-hypopnea index; PLMAI, PLM-related arousal index; REM, rapid eye movement; $\mathrm{SO}_{2}$, oxyhemoglobin saturation; TST, total sleep time. Other abbreviations as in Table 1.

had significantly lower event-free survival than those without severe PLM $(\mathrm{P}=0.002)$. In the univariate Cox proportional hazards regression analysis, greater heart rate (per $1 / \mathrm{min}$ increment; hazard ratio [HR], 1.04; 95\% confidence interval [CI], 1.00-1.07; $\mathrm{P}=0.043$ ), greater NYHA class (per 1 class increment; HR, $1.67 ; 95 \% \mathrm{CI}, 0.96-2.91 ; \mathrm{P}=0.070$ ), lower hemoglobin (per $1 \mathrm{~g} / \mathrm{dL}$ increase; HR, 0.82; 95\% CI, 0.70
0.97; $\mathrm{P}=0.019$ ), lower eGFR (per $1 \mathrm{~mL} / \mathrm{min} / 1.73 \mathrm{~m}^{2}$ increment; HR, 0.98; 95\% CI, 0.97-0.99; $\mathrm{P}=0.019$ ), use of loop diuretics (HR, 6.04; 95\% CI, 0.82-44.4; $\mathrm{P}=0.077$ ), less REM sleep percentage ( $1 \%$ increment; HR, $0.94 ; 95 \%$ CI, $0.89-0.99 ; \mathrm{P}=0.027)$, greater natural log-transformed $\mathrm{AHI}$ (per 1 increment; HR, 1.80; 95\% CI, 1.16-2.81; $\mathrm{P}=0.009$ ), initiation of SDB treatment (HR, 0.21; 95\% CI, 0.05-0.90; 


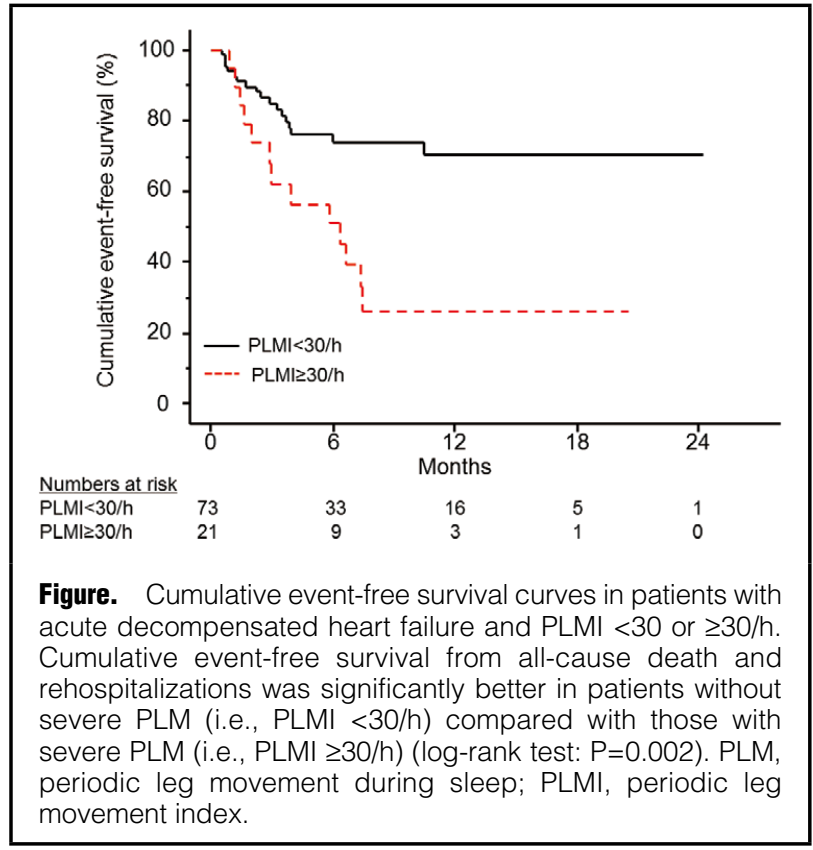

$\mathrm{P}=0.036)$, and the presence of severe PLM (HR, 3.04; $95 \%$ CI, 1.48-6.28; $\mathrm{P}=0.003)$ showed values with $\mathrm{P}<0.10$ and were included in the multivariable analysis (Table 3). The final multivariate forward stepwise Cox proportional hazards regression analysis model is also shown in Table 3. The presence of severe PLM was significantly associated with poor clinical outcomes, together with lower hemoglobin levels and a greater log-transformed AHI.

\section{Discussion}

Our findings provide novel insights into the clinical importance of PLM in patients with HF. First, we observed severe PLM defined as PLMI $\geq 30 / \mathrm{h}$ in $22 \%$ of unselected hospitalized patients following ADHF in association with reduced LVEF. Second, such severe PLM in hospitalized patients following ADHF was associated with a 2.2-fold greater risk of rehospitalization and/or all-cause death compared with patients without severe PLM after adjustment for other significant variables. In addition, this relationship between severe PLM and clinical outcomes remained significant even in the multivariable analysis, including severity of SDB, which is a common sleep-related comorbidity of HF. These findings suggest that identification of severe PLM following an ADHF episode could be an important strategy in comprehensive HF care.

In 4 previous studies among HF patients with reduced LVEF, PLM was generally prevalent. ${ }^{11-14}$ However, the reported prevalence is highly variable ranging, from $19 \%$ to $52 \%$. One possible explanation for this discrepancy may be differences in the severity of HF. The oldest study by Hanly and Zuberi-Khokhar ${ }^{11}$ reported that $52 \%$ of $\mathrm{HF}$ patients with reduced LVEF had moderate to severe PLM, defined as PLMI $\geq 25 / \mathrm{h}$, which is the highest prevalence rate of PLM among the 4 studies. They reported that their $\mathrm{HF}$ patients were stable, but the mean LVEF was $23 \%$, and only NYHA functional class III and IV heart disease was observed, suggesting that the enrolled patients with severely impaired LVEF and highly symptomatic HF may not have really been stable. Three other studies ${ }^{12-14}$ consistently reported a similar prevalence (i.e., 19\%,20\% and $37 \%$ ) even while using a much lower cutoff point for PLMI: $\geq 5 / \mathrm{h}$. Even among those studies, the study by Yumino and colleagues in which some patients with severely impaired LVEF and with NYHA class IV symptoms were enrolled showed a relatively greater prevalence of PLM (i.e., $37 \%)^{14}$ compared with the other 2 studies $(19 \%$ and $20 \%)^{12,13}$ On the other hand, a more recent study by Yoshihisa and colleagues in which patients with either reduced or preserved LVEF and those with variable NYHA class (i.e., class I-IV) disease were enrolled showed that the prevalence of severe PLM, defined as PLMI $\geq 30 / h$, was $16 \%$; this prevalence is less than that of severe PLM in the present study, in which only patients with reduced LVEF and more symptomatic patients following an episode of ADHF were enrolled. If fact, a recent study by Yoshihisa and colleagues reported that patients with severe PLM have impaired exercise capacity. ${ }^{15}$ In addition, in the present study, patients with severe PLM had a worse NYHA functional class and higher CRP and BNP levels than those without severe PLM. Taken together, these findings suggest that patients with more severe HF or those in an unstable state are likely to have severe PLM, and the presence of severe PLM may be an important indicator of such patients' status following ADHF.

A retrospective study in which 29 patients with renal

\begin{tabular}{|c|c|c|c|c|}
\hline \multirow{2}{*}{ Variable } & \multicolumn{2}{|c|}{ Univariable } & \multicolumn{2}{|c|}{ Multivariable } \\
\hline & HR $(95 \% \mathrm{Cl})$ & $P$ value & HR (95\% Cl) & $P$ value \\
\hline Heart rate ( $1 / \mathrm{min}$ increase) & $1.04(1.00-1.07)$ & 0.043 & - & - \\
\hline NYHA class (1 increase) & $1.67(0.96-2.91)$ & 0.070 & - & - \\
\hline Hemoglobin ( $1 \mathrm{~g} / \mathrm{dL}$ increase) & $0.82(0.70-0.97)$ & 0.019 & $0.81(0.68-0.98)$ & 0.028 \\
\hline eGFR ( $1 \mathrm{~mL} / \mathrm{min} / 1.73 \mathrm{~m}^{2}$ increase) & $0.98(0.97-0.99)$ & 0.019 & - & - \\
\hline Use of diuretics-yes & $6.04(0.82-44.4)$ & 0.077 & - & - \\
\hline$\%$ of REM sleep ( $1 \%$ increase) & $0.94(0.89-0.99)$ & 0.027 & - & - \\
\hline Natural log-transformed AHI & $1.80(1.16-2.81)$ & 0.009 & $1.79(1.15-2.79)$ & 0.011 \\
\hline Initiation of SDB treatment-yes & $0.21(0.05-0.90)$ & 0.036 & - & - \\
\hline PLMI $\geq 30 / h$-yes & $3.04(1.48-6.28)$ & 0.003 & $2.16(1.03-4.54)$ & 0.042 \\
\hline
\end{tabular}

$\mathrm{Cl}$, confidence interval; SDB, sleep-disordered breathing. Other abbreviations as in Tables 1,2. 
failure were included reported that the presence of PLM was associated with increased mortality rate. ${ }^{20}$ In another retrospective study in which the polysomnographic, echocardiographic, and outcome data of 584 patients referred because of restless leg syndrome were analyzed, severe PLM defined as PLMI $\geq 35 / \mathrm{h}$ was an independent predictor of severe LV hypertrophy and was associated with increased cardiovascular morbidity and mortality. ${ }^{21}$ In a large observational cohort study, greater severity of PLM was associated with incident cardiovascular disease, particularly peripheral artery disease, in community-dwelling elderly men. ${ }^{22}$ However, the significance of PLM on clinical outcomes in the field of secondary prevention of cardiovascular disease remains to be elucidated. Nevertheless, there are 2 studies in which the relationship between the presence of PLM and prognosis among patients with HF was investigated. Yumino and colleagues reported that in chronic HF patients with reduced LVEF, the presence of PLM, defined as PLMI $\geq 5 / h$, was associated with increased risk of all-cause death. ${ }^{14}$ Furthermore, a study by Yoshihisa and colleagues in which HF patients with either reduced or preserved LVEF and those with a variable NYHA class (i.e., class I-IV) were enrolled showed a significant relationship between severe PLM and increased risk of cardiac death. ${ }^{15}$ There are no previous data regarding the prognostic significance of PLM in hospitalized patients following ADHF in association with reduced LVEF. Therefore, our study is the first to show that severe PLM in hospitalized patients following ADHF in association with reduced LVEF correlated with an increased risk of rehospitalization and/or all-cause death. Results of the present study indicated that identification of severe PLM even in hospitalized patients following ADHF was important to stratify patients with a high risk for subsequent rehospitalization and/or death.

The mechanisms linking PLM and adverse clinical outcomes in patients with HF remain to be elucidated. The most plausible mechanism is the activation of the sympathetic nervous system. Each episode of PLM causes elevations in $\mathrm{BP}$ and heart rate $\mathbf{2 3 , 2 4}^{2}$ through direct and indirect (via arousal) activation of the sympathetic nervous system..$^{25,26}$ Furthermore, several studies have shown the presence of sustained elevation of BP in association with PLM.19,27,28 Such elevations in BP and heart rate during sleep and daytime hypertension in association with PLM may contribute to the LV hypertrophy and increased cardiovascular morbidity and mortality in patients with renal failure, restless leg syndrome, or in community-dwelling elderly men..$^{20-22}$ As well, in a large observational cohort there was a significant association between PLM and cardiac arrhythmias in a subset of community-dwelling elderly men with HF or myocardial infarction. ${ }^{29}$ In patients with HF, PLM-related hemodynamic changes and possibly cardiac arrhythmias through activation of the sympathetic nervous system may adversely affect cardiac load and function to a greater extent than those without HF and lead to not only increased mortality but also increased rehospitalization rates. Other factors associated with PLM, such as inflammation, may also contribute to the worsening of $\mathrm{HF}$ and increased mortality rate. ${ }^{30,31}$ In fact, in the present study, patients with severe PLM showed greater CRP levels than those without severe PLM.

Nevertheless, a cause and effect relationship between the presence of PLM and increased risk of rehospitalization and/or death in patients with HF has not been established from the results of the present study or other previous studies. An activated sympathetic nervous system in association with $\mathrm{HF}$ itself may facilitate the development of PLM. ${ }^{32}$ Furthermore, there is a possibility that PLM is just an indicator of more impaired cardiac function or unstable HF status. Thus, we cannot determine whether severe PLM in hospitalized patients following ADHF is a therapeutic target or not. Although it is reported that dopamine agonists can alleviate PLM in some subjects, ${ }^{31}$ and specific treatment of PLM by dopamine agonists can be effective in reducing the amplitude of heart rate variability and BP elevation induced by PLM, $, 33,34$ there is no evidence regarding the cardiovascular effects of PLM-targeted therapy, especially in patients with HF. Thus, further interventional studies to assess the cardiovascular effects of PLM-targeted therapy in hospitalized patients with HF are needed to investigate the cause and effect relationship between PLM and cardiovascular morbidity and mortality in HF patients.

\section{Study Limitations}

First, the number of subjects was relatively small. Second, although we took confounders into account, we cannot exclude the possibility that unmeasured factors may have explained some of our findings. Third, although activation of the sympathetic nervous system may play a key role in the relationship between severe PLM and poor clinical outcome, we did not assess sympathetic nervous system activity in patients with HF. Finally, we did not assess for the presence of restless leg syndrome symptoms. Thus, the results of the present study should be viewed as preliminary, and further studies with a larger sample size are needed.

In conclusion, results of the present study shed light on the clinical significance of PLM in patients with HF, suggesting that in hospitalized patients following ADHF in association with reduced LVEF, the presence of severe PLM is associated with increased risk of rehospitalization and/or all-cause death, independent of the severity of SDB.

\section{Funding Sources}

This study was partly supported by a Grant-in-Aid for Scientific Research (C) [Grant Number 26507010] and a grant to the Respiratory Failure Research Group from the Ministry of Health, Labor and Welfare, Japan. These funding sources do not have any other roles in this study.

\section{Disclosures}

T. Kasai, S.S., H.M., N.S., M.K. and F.K. are affiliated with a department endowed by Philips Respironics, ResMed, Teijin Home Healthcare, and Fukuda Denshi. H.D. received manuscript fees, research funds, and scholarship funds from Kirin Co. Ltd., Kaken Pharmaceutical Co., Ltd., Abbott Japan Co., Ltd., Astellas Pharma Inc., Astrazeneca K.K., Bayer Yakuhin, Ltd., Boston Scientific Japan K.K., Bristol-Myers Squibb, Daiichi Sankyo Company, MSD K.K., Pfizer Inc., Philips Respironics, Sanofi K.K., and Takeda Pharmaceutical Co. Ltd. The other authors report no conflicts of interest.

\section{References}

1. Hunt SA, Abraham WT, Chin MH, Feldman AM, Francis GS, Ganiats TG, et al. 2009 Focused update incorporated into the ACC/AHA 2005 Guidelines for the Diagnosis and Management of Heart Failure in Adults: A Report of the American College of Cardiology Foundation/American Heart Association Task Force on Practice Guidelines Developed in Collaboration With the International Society for Heart and Lung Transplantation. $J$ Am Coll Cardiol 2009; 53: e1-e90.

2. Januzzi JL Jr, Rehman S, Mueller T, van Kimmenade RR, Lloyd-Jones DM. Importance of biomarkers for long-term mortality prediction in acutely dyspneic patients. Clin Chem 2010; 56: $1814-1821$. 
3. Kattel S, Kasai T, Matsumoto H, Yatsu S, Murata A, Kato T, et al. Association between elevated blood glucose level on admission and long-term mortality in patients with acute decompensated heart failure. J Cardiol 2017; 69: 619-624.

4. Gheorghiade M, De Luca L, Fonarow GC, Filippatos G, Metra M, Francis GS. Pathophysiologic targets in the early phase of acute heart failure syndromes. Am J Cardiol 2005; 96: 11g-17g.

5. Gheorghiade M, Vaduganathan M, Fonarow GC, Bonow RO. Rehospitalization for heart failure: Problems and perspectives. $J$ Am Coll Cardiol 2013; 61: 391-403.

6. McAlister FA, Stewart S, Ferrua S, McMurray JJ. Multidisciplinary strategies for the management of heart failure patients at high risk for admission: A systematic review of randomized trials. $J$ Am Coll Cardiol 2004; 44: 810-819.

7. Fonarow GC, Abraham WT, Albert NM, Stough WG, Gheorghiade M, Greenberg BH, et al. Association between performance measures and clinical outcomes for patients hospitalized with heart failure. JAMA 2007; 297: 61 -70.

8. Mentz RJ, Kelly JP, von Lueder TG, Voors AA, Lam CS, Cowie MR, et al. Noncardiac comorbidities in heart failure with reduced versus preserved ejection fraction. J Am Coll Cardiol 2014; 64: 2281-2293.

9. Ohayon MM, Roth T. Prevalence of restless legs syndrome and periodic limb movement disorder in the general population. $J$ Psychosom Res 2002; 53: 547-554.

10. Scofield H, Roth T, Drake C. Periodic limb movements during sleep: Population prevalence, clinical correlates, and racial differences. Sleep 2008; 31: 1221-1227.

11. Hanly PJ, Zuberi-Khokhar N. Periodic limb movements during sleep in patients with congestive heart failure. Chest 1996; 109: $1497-1502$

12. Javaheri S. Sleep disorders in systolic heart failure: A prospective study of 100 male patients: The final report. Int J Cardiol 2006; 106: $21-28$.

13. Skomro R, Silva R, Alves R, Figueiredo A, Lorenzi-Filho G. The prevalence and significance of periodic leg movements during sleep in patients with congestive heart failure. Sleep Breath 2009; 13: $43-47$.

14. Yumino D, Wang H, Floras JS, Newton GE, Mak S, Ruttanaumpawan $\mathrm{P}$, et al. Relation of periodic leg movements during sleep and mortality in patients with systolic heart failure. Am J Cardiol 2011; 107: 447-451.

15. Yoshihisa A, Suzuki S, Kanno Y, Takiguchi M, Sato A, Miura $\mathrm{S}$, et al. Prognostic significance of periodic leg movements during sleep in heart failure patients. Int J Cardiol 2016; 212: 11-13.

16. McKee PA, Castelli WP, McNamara PM, Kannel WB. The natural history of congestive heart failure: The Framingham study. N Engl J Med 1971; 285: 1441-1446.

17. Berry RB, Brooks R, Gamaldo CE, Harding SM, Lloyd RM, Marcus CL; Vaughn BV for the American Academy of Sleep Medicine. The AASM manual for the scoring of sleep and associated events: Rules, terminology and technical specifications, version 2.2. http://www.aasmnet.org/ Darien, ILL: American Academy of Sleep Medicine, 2015.

18. Matsuo S, Imai E, Horio M, Yasuda Y, Tomita K, Nitta K, et al. Revised equations for estimated GFR from serum creatinine in Japan. Am J Kidney Dis 2009; 53: 982-992.

19. Koo BB, Sillau S, Dean DA 2nd, Lutsey PL, Redline S. Periodic limb movements during sleep and prevalent hypertension in the multi-ethnic study of atherosclerosis. Hypertension 2015; 65: $70-77$

20. Benz RL, Pressman MR, Hovick ET, Peterson DD. Potential novel predictors of mortality in end-stage renal disease patients with sleep disorders. Am J Kidney Dis 2000; 35: 1052-1060.

21. Mirza M, Shen WK, Sofi A, Jahangir A, Mori N, Tajik AJ, et al. Frequent periodic leg movement during sleep is associated with left ventricular hypertrophy and adverse cardiovascular outcomes. $J$ Am Soc Echocardiogr 2013; 26: 783-790.

22. Koo BB, Blackwell T, Ancoli-Israel S, Stone KL, Stefanick ML, Redline S. Association of incident cardiovascular disease with periodic limb movements during sleep in older men: Outcomes of sleep disorders in older men (MrOS) study. Circulation 2011; 124: $1223-1231$.

23. Siddiqui F, Strus J, Ming X, Lee IA, Chokroverty S, Walters AS Rise of blood pressure with periodic limb movements in sleep and wakefulness. Clin Neurophysiol 2007; 118: $1923-1930$.

24. Pennestri MH, Montplaisir J, Fradette L, Lavigne G, Colombo $\mathrm{R}$, Lanfranchi PA. Blood pressure changes associated with periodic leg movements during sleep in healthy subjects. Sleep Med 2013; 14: $555-561$.

25. Bara-Jimenez W, Aksu M, Graham B, Sato S, Hallett M. Periodic limb movements in sleep: State-dependent excitability of the spinal flexor reflex. Neurology 2000; 54: 1609-1616.

26. Pennestri MH, Montplaisir J, Colombo R, Lavigne G, Lanfranchi PA. Nocturnal blood pressure changes in patients with restless legs syndrome. Neurology 2007; 68: 1213-1218.

27. Espinar-Sierra J, Vela-Bueno A, Luque-Otero M. Periodic leg movements in sleep in essential hypertension. Psychiatry Clin Neurosci 1997; 51: 103-107.

28. Dean DA, Wang R, Jacobs DR, Duprez D, Punjabi NM, Zee PC, et al. A systematic assessment of the association of polysomnographic indices with blood pressure: The Multi-Ethnic Study of Atherosclerosis (MESA). Sleep 2015; 38: 587-596.

29. Koo BB, Mehra R, Blackwell T, Ancoli-Israel S, Stone KL, Redline S. Periodic limb movements during sleep and cardiac arrhythmia in older men (MrOS sleep). J Clin Sleep Med 2014; 10: $7-11$.

30. Trotti LM, Rye DB, De Staercke C, Hooper WC, Quyyumi A, Bliwise DL. Elevated C-reactive protein is associated with severe periodic leg movements of sleep in patients with restless legs syndrome. Brain Behav Immun 2012; 26: 1239-1243.

31. Nannapaneni S, Ramar K. Periodic limb movements during sleep and their effect on the cardiovascular system: Is there a final answer? Sleep Med 2014; 15: 379-384.

32. Guggisberg AG, Hess CW, Mathis J. The significance of the sympathetic nervous system in the pathophysiology of periodic leg movements in sleep. Sleep 2007; 30: 755-766.

33. Manconi M, Ferri R, Zucconi M, Clemens S, Rundo F, Oldani A, et al. Effects of acute dopamine-agonist treatment in restless legs syndrome on heart rate variability during sleep. Sleep Med 2011; 12: 47-55.

34. Bauer A, Cassel W, Benes H, Kesper K, Rye D, Sica D, et al. Rotigotine's effect on PLM-associated blood pressure elevations in restless legs syndrome: An RCT. Neurology 2016; 86: 17851793. 